\title{
LONE AURICULAR FIBRILLATION
}

\author{
BY \\ WILLIAM EVANS AND PETER SWANN
}

From the Cardiac Department of the London Hospital

Receivcd December 22, 1953

Mitral stenosis, thyroid toxæmia, cardiac infarction, constrictive pericarditis, and hypertension are among the common causes of auricular fibrillation. Occasionally, however, the arrhythmia is discovered fortuitously and subsequent investigation shows that structural heart disease is absent. The condition has' been described variously as benign, idiopathic, arteriosclerotic, functional, and senile fibrillation, fibrillation of unknown origin and fibrillation without heart disease. We have proposed for it the term lone auricular fibrillation.

Twenty patients were selected for an analysis of the special features that characterize lone fibrillation. Apart from taking each patient's history and recording the findings of a routine clinical examination, special tests were made when necessary to exclude heart disease of any kind. An electrocardiogram was always taken, not so much to confirm the nature of the arrhythmia as to show the absence of preponderance of either ventricle and of cardiac infarction. Once, the basal metabolic rate was estimated and a radio-active iodine test used to exclude thyroid toxæmia. A phonocardiogram was recorded in most of the patients to prove the absence of murmurs and added sounds, especially the mitral snap, even though they had been inaudible. Cardioscopy was carried out in each case to ensure the absence of cardiac enlargement from any source, and particularly to show that mitral valve disease was not the cause of the fibrillation.

Incidence. Although this form of arrhythmia is uncommon when compared with that found in mitral stenosis, it is not rare. We have omitted an estimate of its incidence among the other causes of fibrillation because such a figure is unlikely to be a true one since the condition, through absence of symptoms, often remains undiscovered until a medical examination brings it to light. Those who have written on auricular fibrillation without heart disease (Fowler and Baldridge, 1930; Mohler and Lintgen, 1931; Friedlander and Levine, 1934; Orgain et al, 1936) say that some 6 per cent of all instances of auricular fibrillation occur in healthy subjects, but this estimate included patients with paroxysmal fibrillation except in a paper by Campbell (1935). Hanson and Rutledge (1949) included 20 examples of the paroxysmal kind among 30 cases of fibrillation without heart disease while 46 of the 49 patients reported by Orgain et al. (1936) were instances of paroxysmal fibrillation. We have not included any patient with paroxysmal auricular fibrillation in our series in the belief that it is a separate entity. Such cases may have a different ætiology; Parkinson and Campbell (1930) found no associated heart disease in 15 per cent of their 200 cases of paroxysmal fibrillation. Certainly, the management of the patients in each group is different, for instance those with the paroxysmal variety require continuous digitalization to ensure that the high heart rate, customarily present in the attack, is reduced in order to protect the patient from distressing palpitation. In all our cases the arrhythmia appeared as the established form from the start.

Past History. It happened that a history of rheumatic fever and chorea could not be elicited in any of our patients, but neither the absence nor the presence of such a history should influence decision on the diagnosis of lone fibrillation. In one patient, aged 70 , the arrhythmia appeared 
soon after diphtheria 35 years before and the patient was insistent that it was diphtheria that had caused it.

A family history of the condition was not mentioned by our patients and interrogation about this did not prove informative, and in any event we regarded the patient's version as unreliable evidence of the familial nature of the complaint. Certainly none seemed definite enough to warrant a closer attention to this for none of the relatives examined showed any arrhythmia other than extrasystoles.

Symptoms. Many of the patients in this group were free from symptoms and the arrhythmia was found at a medical examination conducted for reasons unconnected with the heart. In none of the patients were there severe symptoms, and complaints were often only elicited by direct questioning. Shortness of breath was sometimes present, but it was never the outcome of heart failure and the slight handicap was always explained by ageing, obesity, or commencing emphysema. Cardiac pain was not met with. Other slight symptoms such as giddiness, if present, were judged to be unrelated to the heart and its abnormal rhythm. The single relevant complaint was palpitation which was present in 11 of the patients during periods when their occupation involved heavy work that induced tachycardia. When the exertion ceased so did the palpitation abate. The symptom was absent in the other 9 patients who followed occupations that did not evoke strenuous physical exercise. The absence of palpitation in so many patients with lone auricular fibrillation and the trivial discomfort that it caused in the remaining patients is explained by the relative infrequency of the heart rate in this condition.

\section{Clinical Signs}

All of our patients were men and this high incidence of the condition among males has been found by other authors. Thus, Friedlander and Levine (1934) found that 21 of their 22 patients were males, while the 10 examples with established fibrillation reported by Hanson and Rutledge (1949) were all men, whereas 9 of their 20 cases of paroxysmal fibrillation were women. There is need to emphasize the importance of this peculiar sex incidence in lone auricular fibrillation and we would hesitate to diagnose the condition in a woman. Whenever the diagnosis appears to apply in a woman it is likely that continued observation of her progress will show that the fibrillation is of the paroxysmal kind.

- The age in our series varied from 38 to 72 and the average was 56 years. Six were under the age of 50 , eight over 60 , and two over 70 . When the onset of palpitation is regarded as marking the start of the fibrillation it is probable that the condition actually shows a younger age incidence. The average age of 10 patients reported by Hanson and Rutledge (1949) was 36 years. Thus, although lone fibrillation is usually found in older subjects the condition is not confined to the elderly and for that reason the term senile fibrillation is inappropriate.

Special care was taken to exclude thyroid toxamia in every case for it would seem that this in common with mitral stenosis might be a cryptic cause of the arrhythmia and one that casual examination would not discover. A swelling of the thyroid gland was looked for in the neck, and again on cardioscopy as an extension into the thorax. Such searches proved unproductive in every patient. Once, a natural prominence of the eyes led to the application of the radio-active iodine test with a negative result.

The blood pressure was not raised in any patient and both clinical and cardiographic signs of hypertension were absent.

The relatively slow heart rate in lone auricular fibrillation is a characteristic finding and is chief among the clinical signs that distinguish this form of fibrillation from the others. Thus, in our series it was never as rapid as 100 a minute. In only two cases was it 90 or over during the first clinical examination. It was 70 or under in eleven and 60 or under in three. This slower rate was natural for each patient and not the result of digitalization. Induced exercise often increased the rate sufficiently to cause palpitation and it was in these patients that this symptom presented 
if their occupation was arduous enough to produce this moderate tachycardia. A pulse deficit, if met with in any of the patients, was negligible.

No significant murmurs or added sounds appeared in any of our cases although these were sought in the knowledge that mitral stenosis might be the cause of the fibrillation even though a middiastolic murmur had evaded auscultation. For the same reason a mitral snap sound was listened for attentively. Although clinical examination failed to bring to light any such murmurs or sounds a phonocardiogram was recorded in most instances, so as to specially exclude the auscultatory signs of mitral valve disease (Fig. 1).

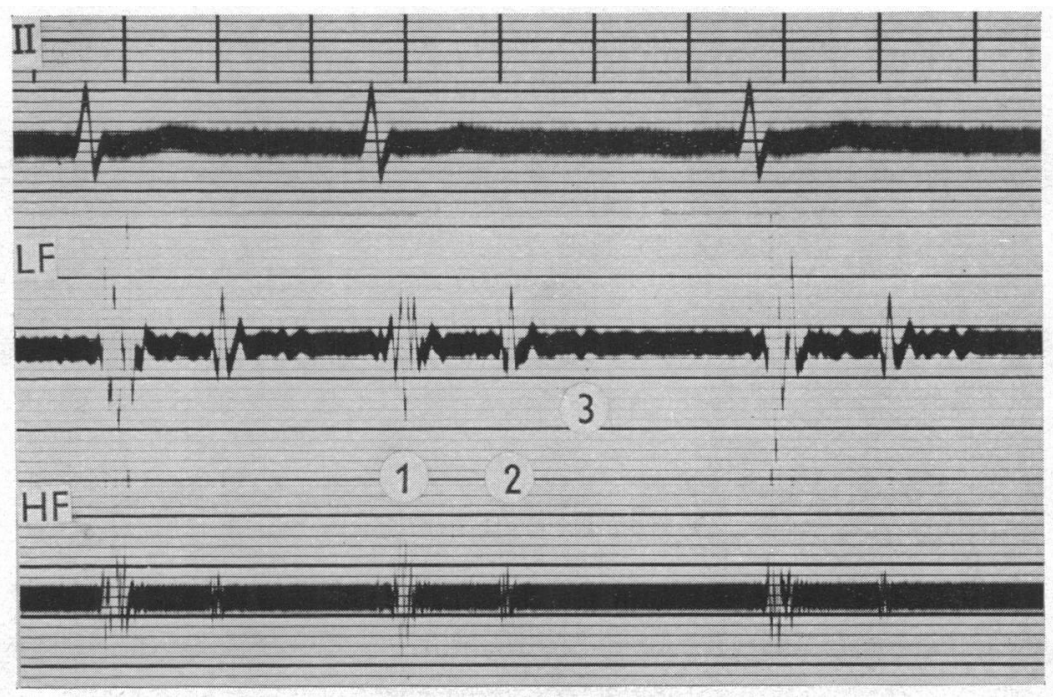

Fig. 1.-Phonocardiogram (low and high frequency) to show the absence of significant added sounds and murmurs. The recorded third heart sound was not heard.

The electrocardiogram (Fig. 2) was recorded in every patient; it always confirmed the presence of auricular fibrillation and the absence of right or left ventricular preponderance. Sometimes it showed the marks of previous digitalization. It is known that it may be difficult to see the effects of cardiac infarction in a tracing that also shows auricular fibrillation. Frank inversion of the $\mathrm{T}$ wave was never present and in those showing a depressed $\mathrm{T}$ wave it was judged to have been caused by digitalis in every case.

The absence of cardiac enlargement was confirmed in each patient during cardioscopy, and inspection of the left auricle was carried out in the oblique positions during a barium swallow, for its enlargement would naturally invite the conclusion that mitral stenosis or, less commonly, thyroid toxæmia had caused the fibrillation. No example of generalized enlargement of the heart nor of left auricular enlargement was found among the 20 patients, nor did this develop in four patients watched over 10 years, nor in two watched over 20 years (Fig. 3 and 4). This experience permits us to state that auricular fibrillation when both heart disease and thyroid toxæmia have been excluded as causes, does not produce enlargement of the left auricle nor of the heart as a whole even when it has persisted continuously in elderly subjects over many years. Indeed, we regard it as conditional for the diagnosis of lone auricular fibrillation that no enlargement of the heart should be present, for such enlargement, even though moderate in degree, indicates that a common cause like mitral stenosis or a less common one like cardiac amyloidosis is present, even if a careful clinical examination fails at the time to identify the lesion. Likewise, radiological examination excluded the presence of pulmonary congestion both at the start and at subsequent inspection through the years. 

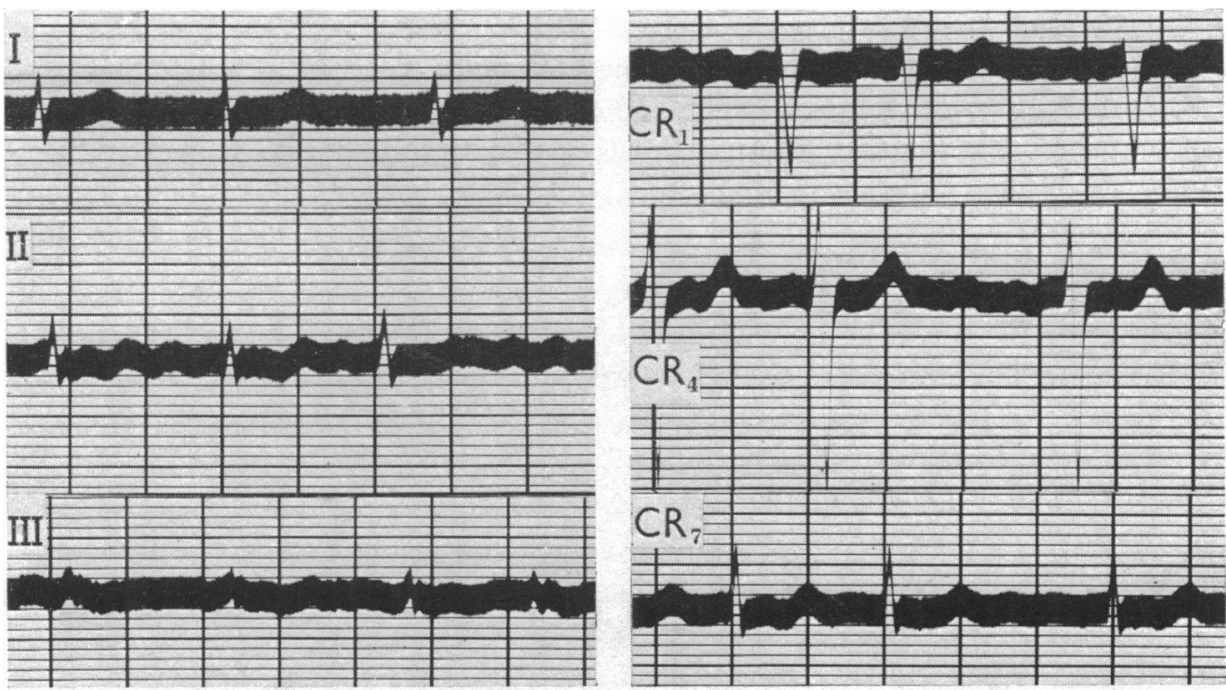

Fig. 2.-Electrocardiogram to show auricular fibrillation and the absence of preponderance of either ventricle. The $T$ waves are upright

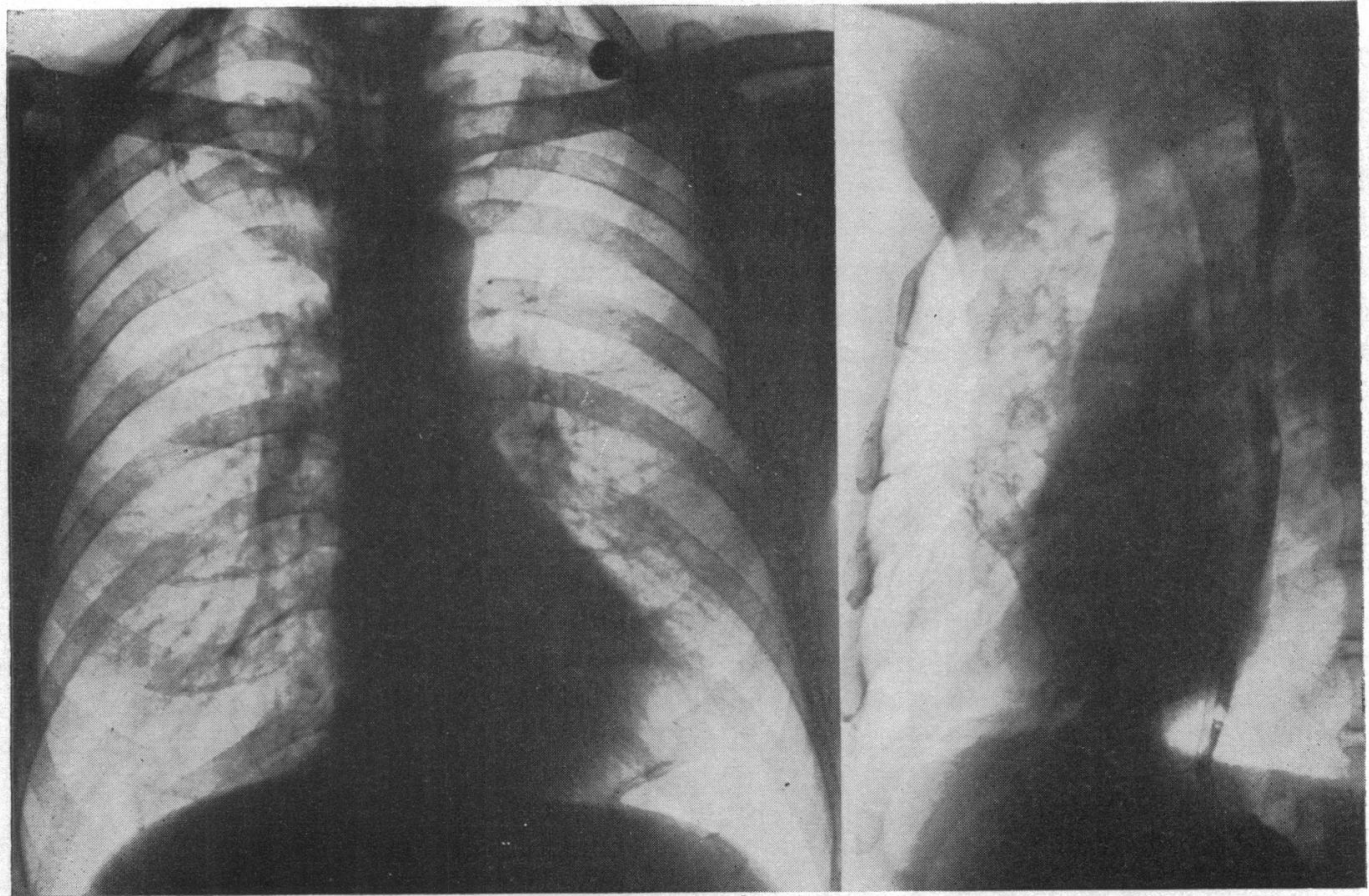

Fig. 3.-Teleradiogram (anterior and left oblique) to show absence of both generalized and left auricular enlargement and of pulmonary congestion. Fibrillation had been present for 15 years. 


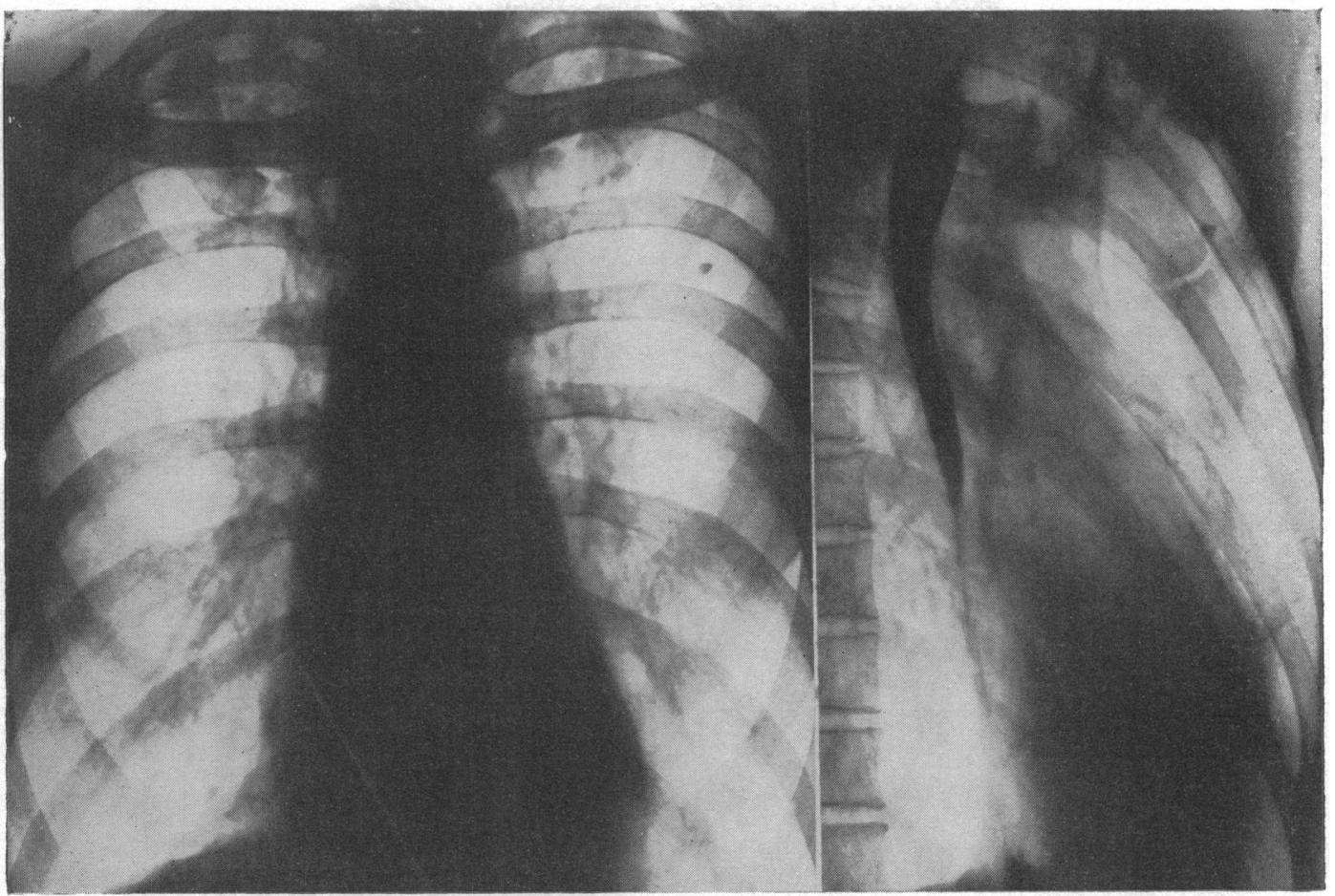

Fig. 4.-Teleradiogram (anterior and right oblique) to show absence of both generalized and left auricular enlargement, and of pulmonary congestion. Fibrillation had been present for 12 years.

\section{Progress AND MANAGement}

The prognosis in our patients proved to be uneventful so that the condition did not jeopardize life in a single instance, and did not even prove a handicap to the majority. No instance of embolism has been met with among the patients and it would appear that lone auricular fibrillation does not favour, and may not be liable to, the complication of intra-cardiac thrombosis. Longevity is unaffected and the condition need never hamper livelihood.

Reassurance should be uppermost in the treatment or management of a patient with lone auricular fibrillation. An urge to re-instate sinus rhythm in an elderly subject should be suppressed in the knowledge that the heart is under no duress from this form of fibrillation; quinidine should not be used. In a patient following a heavy occupation where exertion increases the heart rate sufficiently to cause palpitation, continuous digitalization with one grain of the leaf daily is ideal treatment, and it should be made known to the patient that digitalis therapy is initiated with the purpose of subduing the heart rate and not because of a need to support the heart muscle.

\section{CONCLUSIONS}

When auricular fibrillation has been shown to be taking place in the absence of heart disease or thyroid toxæmia we suggest that the condition be named lone auricular fibrillation.

Patients with paroxysmal fibrillation should not be included in this group in that the arrhythmia probably arises from a separate mechanism and the management of the two conditions is different.

Lone fibrillation is probably not met with in women and all of our 20 patients were men. Although commoner in the older it is sometimes found in younger subjects. 
The condition is often without symptoms and discovered fortuitously in the course of a routine medical examination. Because of the relatively slow basic heart rate, seldom over 90 a minute, palpitation is often absent, and when present it is in a patient following an occupation where periodic exertion induces tachycardia sufficient to cause the symptom. Signs of heart failure are never present. The usual causes of fibrillation like mitral stenosis, thyroid toxæmia, cardiac infarction, constrictive pericarditis, and hypertension, need to be excluded on clinical, cardiographic and cardioscopic examination before the diagnosis of lone auricular fibrillation is substantiated. There are no murmurs or added sounds and the absence of cardiac enlargement is a particular characteristic; not only must generalized enlargement of the heart be excluded by cardioscopy, but distension of the left auricle must be shown to be absent with the aid of a barium swallow. Similarly, cardioscopy should exclude the presence of pulmonary congestion connoting heart failure. If either cardiac enlargement or pulmonary congestion appear in the course of auricular fibrillation it has not been of the lone kind, and the cause of the arrhythmia, though often evasive must be sought.

Prognosis is unaffected by lone auricular fibrillation because embolism, progressive cardiac enlargement, and heart failure do not develop during the many years when it might be the prevailing heart rhythm. Reassurance, therefore, must be uppermost in the management of the case. It is unnecessary to restore normal rhythm. Should habitual exercise connected with the patient's occupation induce a degree of quickening of the heart rate that causes palpitation, continuous digitalization should be given in order to ensure a slow heart rate on such occasions; at the same time the patient should be informed of the purpose of such therapy so that any suspicion that it is given to support a failing heart is removed.

We wish to acknowledge help given by Dr. J. Stobo Prichard who supervised the progress of the earlier cases when he was Registrar to the Cardiac Department.

\section{REFERENCES}

Campbell, M. (1935). Guy's Hosp. Rep., 85, 471.

Fowler, W. M., and Baldridge, C. W. (1930). Amer. Heart J., 6, 183.

Friedlander, R. D., and Levine, S. A. (1934). New Eng. J. Med., 211, 624.

Hanson, H. M., and Rutledge, D. I. (1949). New Eng. J. Med., $240,947$.

Mohler, H. K., and Lintgen, C. (1931). Pennsylvania med. J., 35, 68.

Orgain, E. S., Wolff, L., and White, P. D. (1936). Arch. intern. Med., 57, 493.

Parkinson, J., and Campbell, M. (1930). Quart. J. Med., 23, 67. 